\title{
VALIDATION OF MICROSCOPIC DYNAMICS OF GROUPING PEDESTRIANS BEHAVIOR: FROM OBSERVATION TO MODELING AND SIMULATION
}

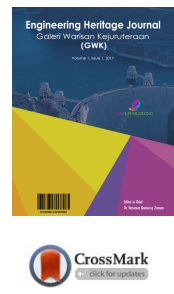

\author{
Noorhazlinda Abd Rahman ${ }^{1 *}$, Herni Halim ${ }^{1}$, Hitoshi Gotoh ${ }^{2}$, Eiji Harada² \\ ${ }^{1}$ School of Civil Engineering, Engineering Campus, Universiti Sains Malaysia, 14300 Nibong Tebal, Penang, Malaysia. \\ ${ }^{2}$ Department of Civil and Earth Resources Engineering, Graduate School of Engineering, Katsura Campus, Kyoto University, \\ Nishikyo-ku, 615-8540, Kyoto, Japan. \\ *Corresponding author email: celindarahman@usm.my
}

This is an open access article distributed under the Creative Commons Attribution License, which permits unrestricted use, distribution, and reproduction in any medium, provided the original work is properly cited

\section{ARTICLE DETAILS}

\section{Article history:}

Received 25 August 2017

Accepted 08 October 2017

Available online 2 November 2017

Keywords:

pedestrian, grouping behavior, simulation, validation, DEM-based model

\begin{abstract}
A B S T R A C T
The aim of this paper is to highlight the role of computer-based study in modeling and simulating the dynamical behavior of pedestrians grouping behavior or a phenomenon of pedestrians walk in group due to social ties. For the purpose of modeling and simulation validation, the results of field observations were reported in this paper. The existing simulator, namely Distinct Element Method (DEM)-based Multi-agent model (DEM-based model) was utilized and modified in this study. The modification was made by introducing two new terms of interaction forces which define the grouping behavior. Validation on the modified DEM-based model (DEM-based with grouping behavior model) was performed in two phases; (1) looking for suitable grouping scenarios from the footage of observations conducted; and (2) simulating suitable scenarios in (1). It shows that DEM-based with grouping behavior model can realistically simulate grouping behavior in a crowd.
\end{abstract}

\section{INTRODUCTION}

Recent data on the urbanization in Malaysia revealed that the number of people living in urban areas has increased from $65.69 \%$ in 2004 to $74.71 \%$ in 2015 [1]. Facing this trend, developing urban sustainable transportation strategies becomes an important necessity for the government to improve the quality of life of the citizens. Currently, urban planning activities are centering on walkability to look into how conducive and friendly the urban environment is for walking (e.g., quality of sidewalks, street connectivity, safety of pedestrian crossings, access to buildings and transit facilities, etc.). Hence, the understanding of crowd dynamics while walking and the acquisition of empirical evidences about pedestrian walking behavior in architectural buildings and any built environment is important for the purpose of understanding the pedestrian flow and model validation.

Study on modeling and visualizing the dynamics of pedestrian crowd behavior, especially in urban environment is relatively new in Malaysia. Within the above scenarios, the role of computer-based systems in modeling and simulating the dynamical behavior of pedestrians is less discussed despite the fact that this medium provides significant advantages in investigating the dynamics of pedestrian's behavior in an artificial way, which is often either not possible or too risky in the real world. Hence, this paper presents the results of conducted field observations focus on grouping pedestrian behavior in urban contexts, for modeling and simulation validation.

\subsection{Grouping Behavior in a Crowd and Its Replication}

Grouping is a common phenomenon that emerges in a crowd. Most individuals walk in groups due to social ties. Observations made by [2] resulted that the percentage of observed pedestrians walk in groups in a commercial street was about $70 \%$ with the most frequent size of grouping is two to four members.

The groups of persons (or grouping) have a significant effect on the flow of the crowd. [3] stated that group of individuals typically moves slower than a single person and acts as a virtual barrier and hence, slows down the crowd. As discussed by [4], a slow movement can be resulted from different group structures and consequently affect evacuation efficiency in emergency situations. [2] extended the social force model by incorporating social interactions among people walking in groups and discussed its impact on the crowd dynamics.

The modeling of grouping pedestrian can lead to a more representative crowd behavior, however, less study conducted to confirm the reliability of the model. This fundamental study was carried out to model grouping behavior together with the model validation. Observations and video footages of public area had been conducted as a basis of grouping behavior model development and validation work.

Part of the methodology in this study was observation of the crowd using video footage at public location. In this contribution, the existing DEMbased simulator $[5,6]$ was modified by introducing grouping behavior model. This grouping behavior model was developed in relation to individual basis of person behavior that describes how a person interacts with the same group's persons and different groups' persons. The validation between simulated scenarios and the real situation were conducted by taking video image as references and comparison were made with the simulated grouping behavior produced by the new developed model.

\section{MATERIAL AND METHOD}

\subsection{Video Footage}

The first part of this study involves video footage activities. Two different locations had been selected. The first footage was shot at the pathway near a riverbank (location A - Figure 1 (a)). And, the second footage was shot at the pathway connecting a shopping mall and a store, near the train station (location B - Figure 1 (b)). Both locations have long pathways which enable to view persons walk for a sufficient length of time and subsequently investigate their walking behavior. Each location has different types of crowd activities, for instance, sightseeing at location $\mathrm{A}$ and commuting/traveling at location B. The footages were taken in four different Sundays, but during similar time zone in the afternoon around two o'clock. Two o'clock was an appropriate time whereby the chosen area full of people outing and sightseeing. Video footages were then analyzed in the laboratory. The frequency distribution of grouping size observed in a crowd and the average walking velocity of grouping pedestrians were computed. Table 1 summarizes total number of groups observed.

\subsection{Grouping Behavior Model}

The development of the grouping behavior model was based on the postulation of the motion of the pedestrian groups in a crowd. And, the motion of pedestrian was postulated based on local interactions in a crowd. Local interactions were model from two aspects, intra-group and inter-group interactions. The intra-group interaction refers to the interaction among persons who belong to the same group, while the inter-group interaction refers to the interaction of a person with other 
persons in different groups and with

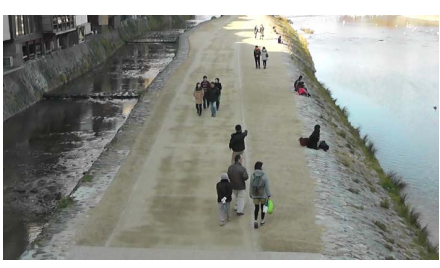

(a) Location $\mathbf{A}$ - the pathway at the riverbank

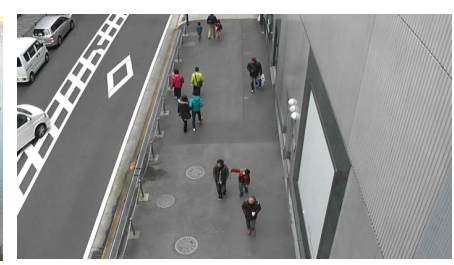

(b) Location $\mathbf{B}$ - the pathway connecting a shopping mall and a store, near the train station
Figure 1: Location of Video Footage

Table 1: Total number of groups observed

\begin{tabular}{|c|l|c|}
\hline Location & Day & $\begin{array}{c}\text { No. of Group } \\
\text { Observed }\end{array}$ \\
\hline \multirow{2}{*}{ A } & Day 1: Sunday & 50 \\
\cline { 2 - 3 } & Day 2: Sunday & 35 \\
\hline \multirow{2}{*}{ B } & Day 1: Sunday & 364 \\
\cline { 2 - 3 } & Day 2: Sunday & 387 \\
\hline Total & & $\mathbf{8 3 6}$ \\
\hline
\end{tabular}

other physical obstacles. The existing simulator, DEM-based model, is modified to include these two new terms of interactions.

In this modeling, a crowd is considered consists of single persons and groups of persons. An isolated walking person is considered to be a group of itself (single). Each person is governed by the translational and rotational equations of motion in which the motion of each person is described by the vector addition of the separate force terms: an autonomous driving force, $\boldsymbol{F}_{\mathrm{aw}}$ and interaction forces, $\boldsymbol{F}_{\text {int }}$ and a torque, $\boldsymbol{T}_{\text {int }}$. The complete governing equations are described as follows:

$m \frac{\mathrm{d}^{2} \boldsymbol{x}_{\boldsymbol{i}}}{\mathrm{d} t^{2}}=\boldsymbol{F}_{i n t}+\boldsymbol{F}_{a w} ; \boldsymbol{F}_{a w}=m \boldsymbol{a}$

(1)

$I \frac{\mathrm{d} \omega_{i}}{\mathrm{~d} t}=\boldsymbol{T}_{\text {int }}$

where $m$ and $I$ are the mass and moment of inertia of a person respectively. The person is represented as a cylindrical element. $\boldsymbol{x}_{\mathrm{i}}$ is the positional vector of the person $i$ and $\omega_{\mathrm{i}}$ is the angular velocity of the person $i$. $\boldsymbol{a}$ is the acceleration vector. Each person $i$ is assumed to accelerate up to the specific equilibrium velocity, $\boldsymbol{v}_{\text {limit }}$ by the autonomous walking force $\boldsymbol{F}_{\text {aw }}$.

The interaction forces are defined as in Eq. 3;

$\boldsymbol{F}_{\text {int }}=\boldsymbol{F}_{\text {intra }}+\boldsymbol{F}_{\text {inter }}$

where $\boldsymbol{F}_{\text {intra }}$ is the intra-group interaction force acting between a person and his/her relative neighbor in the same group and $\boldsymbol{F}_{\text {inter }}$ is the intergroup interaction force acting between groups and group-physical obstacle element.

In the existing DEM-based model, the interaction force only considers the repulsion effect which defined based on the overlapping between two isolated persons elements or between person element and physical obstacle element. Here the estimation of the intra-group and inter-group interactions forces are made by the Voigt model in reference to the physical contact area introduced for each person to hold the group formation and the perception domain for each group to avoid collision with other groups. Figure 2 illustrates the domain of the interaction.

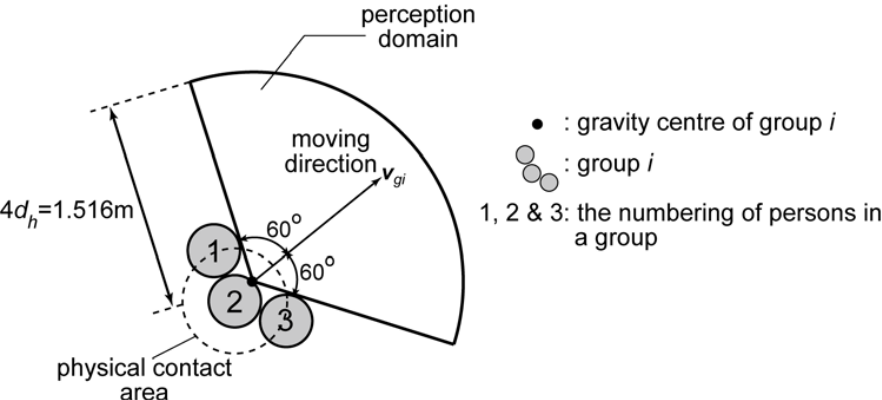

Figure 2: The domain of interaction for intra- and inter-group
To sustain a group formation so that persons who belong to the same group behave cohesively without any splitting in walking, the intragroup interaction forces, $\boldsymbol{F}_{\text {intra }}$ include the attraction and repulsion effects. The attraction effect is formulated to prevent the separation between two-person elements in the same group, and the repulsion effect is to avoid overlap between person elements in the same group. The reference is made based on the relative position of each person in the same group. In case of Fig. 2, the 2 nd person relates with neighboring person of the 1 st and the 3rd.

The whole of the intra-group interaction force acting on the person $\mathrm{i}$ is described as follows:

$\boldsymbol{F}_{\text {intra }}=\boldsymbol{F}_{\text {rep }}+\boldsymbol{F}_{\text {att }}=\sum \mathrm{f}(\neq \mathrm{i}) \boldsymbol{f}_{\text {igrep }}+\sum \mathrm{f}(\neq \mathrm{i}) \boldsymbol{f}_{\text {igatt }}$

where $\boldsymbol{F}_{\text {rep }}$ is the net physical repulsive force and $\boldsymbol{F}_{\text {att }}$ is the net psychological attractive force, $\boldsymbol{f}_{\text {igrep }}$ is the local intra-group physical repulsive force and $\boldsymbol{f}_{\text {igatt }}$ is the local intra-group psychological attractive force.

Each person is having the physical or psychological contact area. The psychological attractive force will be triggered if the relative position between a person $i$ and his/her relative neighbor $j$ is satisfying the condition in Eq. (5). Meanwhile, if the person and his/her relative neighbor are in physical contact, the physical repulsive force is triggered, as in Eq. (6).

$\left|\boldsymbol{x}_{i}-\boldsymbol{x}_{j}\right|>\frac{d_{h i}+d_{h j}}{2}$

$\left|\boldsymbol{x}_{i}-\boldsymbol{x}_{j}\right|<\frac{d_{h i}+d_{h j}}{2}$

where $\boldsymbol{X}_{\mathrm{i}}$ is the positional vector of person $i, \boldsymbol{X}_{\mathrm{j}}$ is the positional vector of relative neighbor ${ }_{j}$ and $d_{\mathrm{h}}$ is the diameter of person $(0.379 \mathrm{~m})$.

\subsection{Inter-group Interaction}

To avoid collision with other groups, the inter-group interaction considers the repulsion effects which defined based on the overlapping between isolated group elements or between group elements and obstacle elements. For this, the formulation is developed by considering a group as a single entity (see Fig. 2). The gravity center of each group $i, \boldsymbol{x}_{\mathrm{gi}}$ is determined by calculating the arithmetic mean of positions of persons in a group $i$, as in Eq. (7). Besides, the mean velocity of each group $i, \boldsymbol{v}_{\mathrm{gi}}$ is determined by finding the arithmetic mean velocity of persons in a group $i$, as in Eq. (8).

$=\frac{1}{n} \sum_{k=1}^{n} \boldsymbol{x}_{k}$ $\boldsymbol{x}_{g i}$

$=\frac{1}{n} \sum_{k=1}^{n} \boldsymbol{v}_{k}$

$\mathcal{v}_{g i}$

where $\boldsymbol{x}_{\mathrm{k}}$ is the positional vector of a person in a group $i, n$ is total number of persons in a group $i$. Each group is having the perception domain with the radius $4 \mathrm{dh}$ and angle of vision $120^{\circ}$

The repulsive forces will only be triggered if Eq. 9 is satisfied;

$\left|\boldsymbol{x}_{\mathrm{gi}}-\boldsymbol{x}_{\mathrm{gj}}\right| \leq 4 d_{\mathrm{h}} ;\left|\boldsymbol{x}_{\mathrm{gi}}-\boldsymbol{x}_{\mathrm{W}}\right| \leq 4 \mathrm{~d}_{\mathrm{W}}$

where $d_{\mathrm{W}}$ is the diameter of the wall $(0.379 \mathrm{~m})$. The interaction between isolated group elements or between group element and wall element is defined as the following;

$\boldsymbol{F}_{\text {inter }}=\boldsymbol{F}_{\text {rep }}\left(\because \boldsymbol{F}_{\text {att }}=0\right)$

$\boldsymbol{F}_{\text {rep }}$ is the net repulsive force between the group $i$ and $j$. The net repulsive forces between groups and group-wall elements are quantified by the summation of the local repulsive forces between groups and group-wall elements.

$\boldsymbol{F}_{\text {rep }}=\boldsymbol{\Sigma} \boldsymbol{f}_{\text {ogrep }}$

where $\boldsymbol{f}_{\text {ogrep }}$ is the local inter-group repulsive force. 


\subsection{Validation Works}

For the purpose of validation of the developed grouping behavior model, two processes were performed. The first process was looking for the suitable scenario from the footages. The footages were manually examined. The criteria for the suitable scenario were contra-flow and involved multiple groups including persons who walk alone. The suitable (chosen) scenario was then saved in sequence images of PNG format. Afterwards, the number of groups, the size of groups and the average walking velocity of each group were determined.

In the second process, simulation of the chosen scenario was performed by employing the newly developed model, DEM-based with group behavior model and the results of simulation were then compared with the footage of chosen scenario.

\section{RESULTS AND DISCUSSION}

\subsection{The Results of Field Observations}

There were 162 people at location A and 1163 people at location B observed. A total number of groups observed were 836 groups. The proportion of people who belongs to a group is $67.1 \%$ at location A and $42.1 \%$ at location B. Table 2 and Fig. 3 show the details of the frequency distribution of grouping size observed in a crowd. From the results, groups composed of two persons are the most frequent, and the drastic decrease of the frequency distribution in the groups of three and four persons is found without dependence on activities. Additionally, groups of size with five and larger are infrequent. This is in agreement with previous observation [2]. In addition, the average walking velocity of observed persons in groups were also computed and the results are shown in Table 2. From the results, with the increasing size of grouping, the average walking velocity of a grouping pedestrian is decreasing.

Table 2: Frequency distribution of grouping size and the average walking velocity

\begin{tabular}{|ccccccccc|}
\hline \multirow{2}{*}{$\begin{array}{c}\text { Group } \\
\text { size }\end{array}$} & \multicolumn{2}{c}{$\begin{array}{c}\text { No. of } \\
\text { group }\end{array}$} & \multicolumn{2}{c}{ Population } & \multicolumn{2}{c}{$\begin{array}{c}\text { Group } \\
\text { percentage } \\
\text { (\%) }\end{array}$} & $\begin{array}{c}\text { Average } \\
\text { walking } \\
\text { velocity (m/s) }\end{array}$ \\
\cline { 2 - 10 } & $\mathbf{A}$ & $\mathbf{B}$ & $\mathbf{A}$ & $\mathbf{B}$ & $\mathbf{A}$ & $\mathbf{B}$ & $\mathbf{A}$ & $\mathbf{B}$ \\
\hline Single & 28 & 435 & 28 & 435 & 32.9 & 57.9 & 1.19 & 1.21 \\
2 & 45 & 242 & 90 & 484 & 52.9 & 32.2 & 1.15 & 1.17 \\
3 & 6 & 55 & 18 & 165 & 7.1 & 7.3 & 0.95 & 1.02 \\
4 & 4 & 16 & 16 & 64 & 4.7 & 2.1 & 0.86 & 0.86 \\
$>5$ & 2 & 3 & 10 & 15 & 2.4 & 0.4 & 0.80 & 0.70 \\
\hline Total & $\mathbf{8 5}$ & $\mathbf{7 5 1}$ & $\mathbf{1 6 2}$ & $\mathbf{1 1 6 3}$ & $\mathbf{1 0 0 . 0}$ & $\mathbf{1 0 0 . 0}$ & & \\
\hline
\end{tabular}

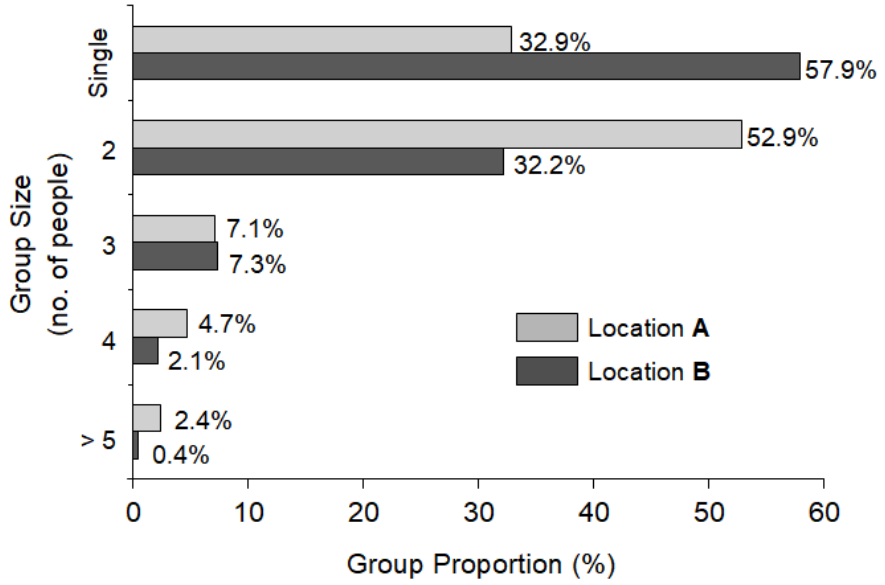

Figure 3: Frequency distribution of grouping size

\subsection{Suitable Scenario from The Footage}

For the purpose of validation work only location B was considered. This was due to the condition of walking pedestrian's activity observed in location B more vibrant than that in location A. The video image at location B was converted into the image sequence of PNG format by using Adobe $\AA$ After Effect CS4 software. After reviewing the entire video footage, the suitable scenario was extracted, and named as chosen scenario. The length of the chosen scenario is $10 \mathrm{~s}$. Then, the image sequence (PNG format) of the chosen scenario was sorted into every $2 \mathrm{~s}$. The initial position of persons in specific group and persons who walk alone were determined using the HBS tool to project the position of persons on a 2D plane. The initial position for chosen scenario is shown in Fig. 3. For validation work, the observation trap is defined and shown in Fig. 3. Fig. 4 (a) shows the perspective view which took from the video footage and Fig. 4 (b) illustrates the schematic diagram of the observation trap. The northward is as shown in the figure. The dimension of the

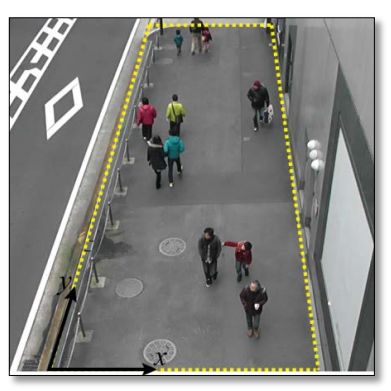

(a). Perspective view)

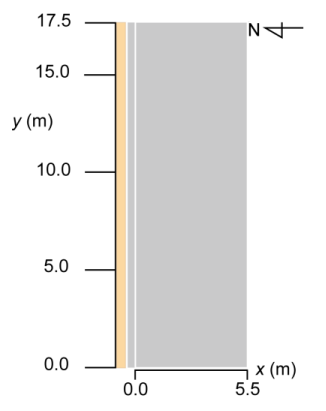

(b). Schematic diagram
Figure 4: Initial position and the observation trap

\subsection{Validation Outcomes}

Basically, two different chosen scenarios had been established, and named as Scenario 1 and Scenario 2. Visually, the motion of persons in the chosen scenarios is shown in Fig. 5 (Scenario 1) and Fig. 6 (Scenario 2 ) by the snapshots. Model 1 is referring to DEM-based model without a grouping behavior model, and Model 2 is for DEM-based model with a grouping behavior model.

From Scenario 1, the unrealistic situations can be seen clearly in Model 1 , particularly at the time $t=6.0 \mathrm{~s}$, where groups G2W and G3W were not maintaining group formation. On the other hand, in Model 2, grouping phenomena were well produced and shows good agreement with the observation. These can be seen in the snapshots, where pedestrians who belong to a group maintains their group formation even though the shape of the group changes. Further analysis was made by plotting the time series of average walking velocity for groups G3W, as shown in Fig. 7. From the graph, the average walking velocity for Model 1 shows high readings compared to Model 2. However, the readings of Model 2 was less than the readings of the observation. This scenario was further analyzed by plotting the time series of relative displacement between members in a group for groups G3W, as shown in Fig. 8. From the graph, it can be seen in Model 1, relative displacement between members in the group were drifting apart along the motion. Meanwhile, for Model 2, the relative displacement between members in the group maintained group formation during the motion. However, when compared to the observation the readings of Model 2 are less. This indirectly affects the reading of average walking velocity. The readings are not having a good agreement with the observation due to footage were performed by a video static camera in the field with a perspective view setting. Ideally, the camera should be set with a top view. Due to site constraints, the camera has to be set with a perspective view to the real-world plane.

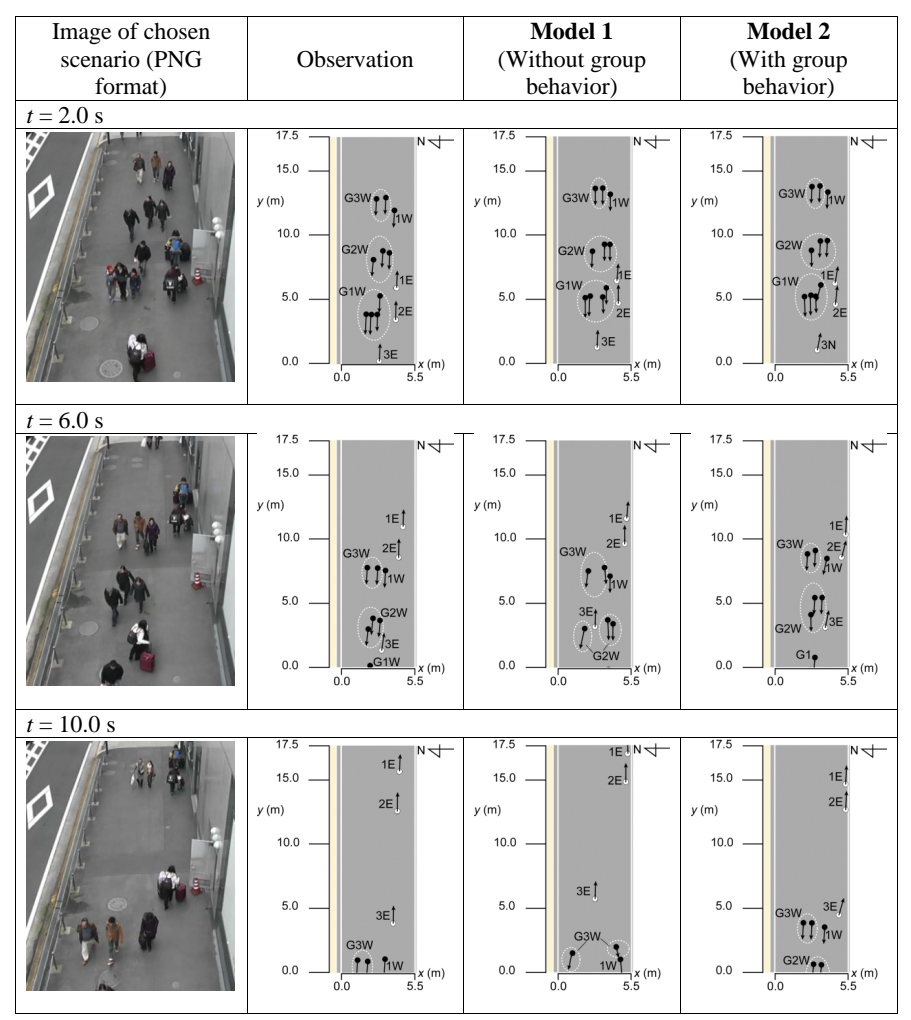

Figure 5: Validation and comparison between Model 1 and Model 2 for Scenario 1 
Similar phenomenon of not maintaining group formation can be found in Scenario 2 for Model 1 (look at groups G1W and G2W at $t=6.0 \mathrm{~s}$ and $t=$ 10.0 s). Meanwhile from Model 2, grouping phenomena were well produced and showed good agreement with observations.

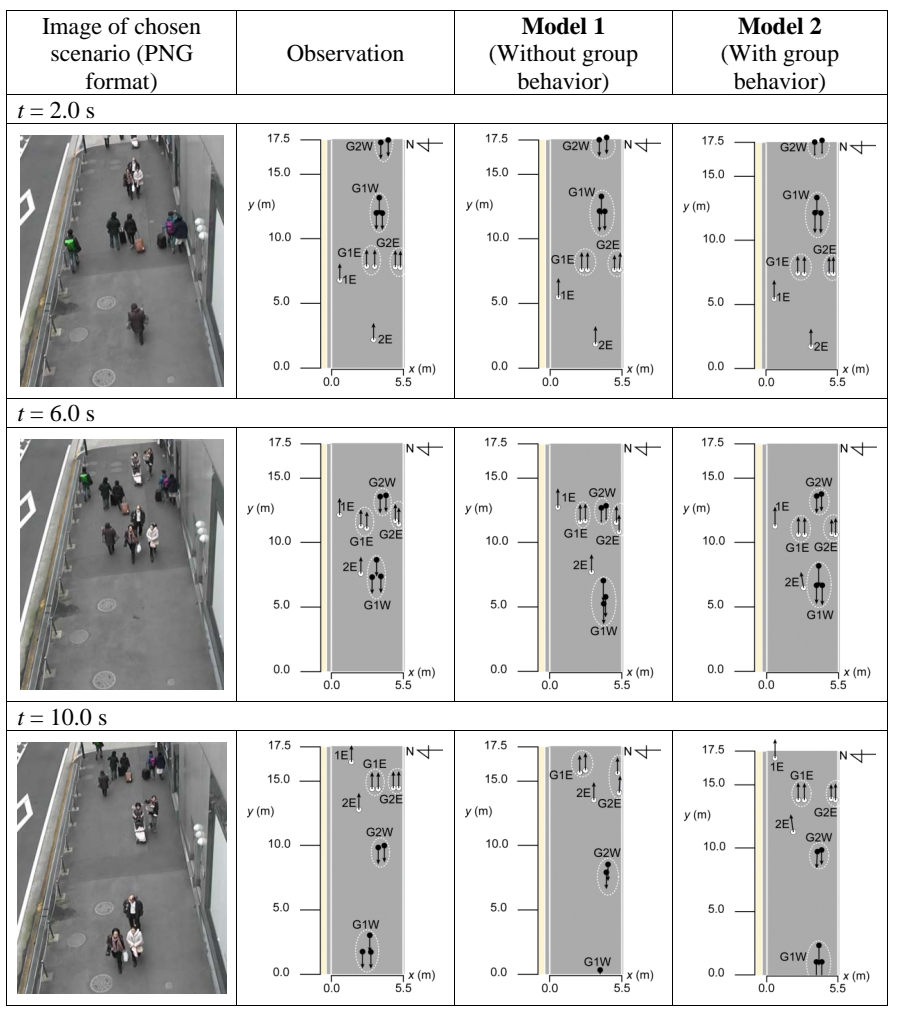

Figure 6: Validation and comparison between Model 1 and Model 2 for Scenario 2

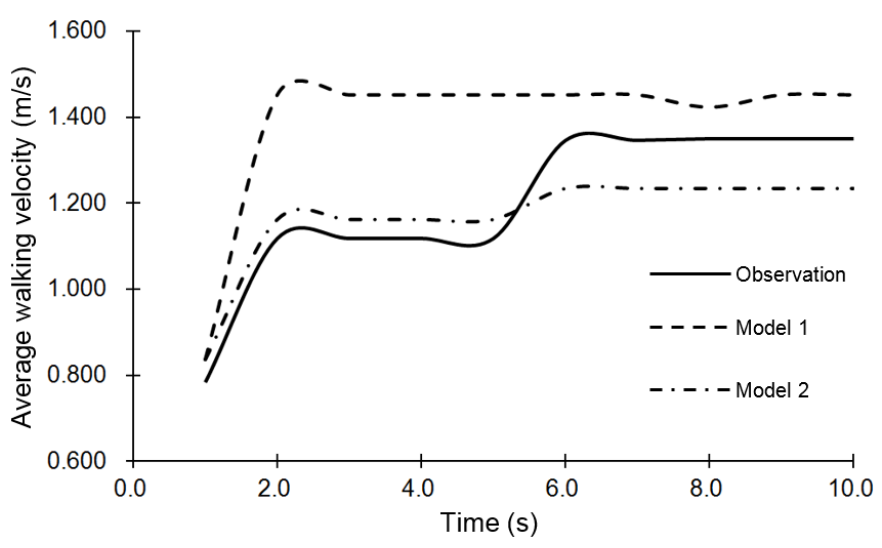

Figure 7: Time series of average walking velocity for G3W for Scenario 1

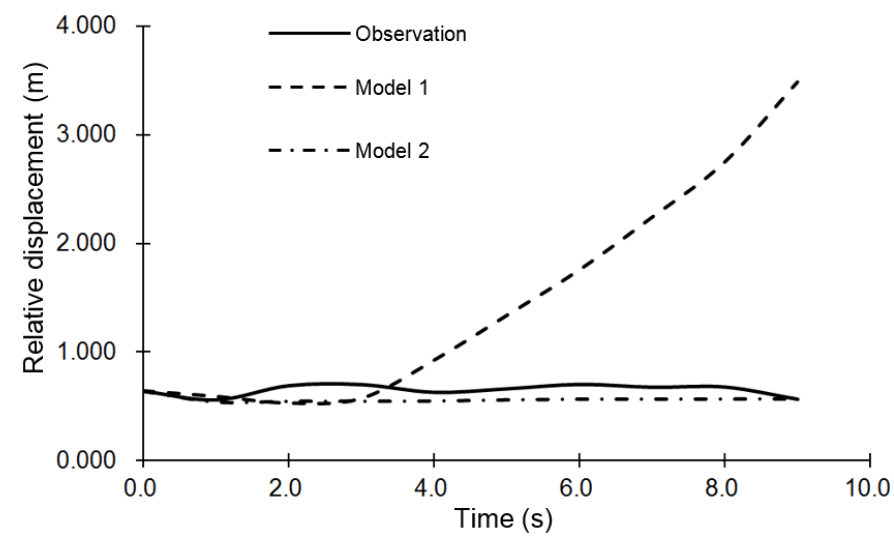

Figure 8: Time series of relative displacement for G3W for Scenario 1

\section{CONCLUSION}

This work is objectively to validate the developed grouping behavior model by comparison with the observations. The existing simulator, DEM-based model, is modified by incorporating grouping behavior model. Previously, DEM-based model was developed based on the

interaction between the isolated individuals which bring significant limitations in simulating grouping behavior as it were found in the validation work. The intra-group and inter-group interactions were introduced in DEM-based model to overcome such limitations. The intra-group interaction was introduced so that persons who belong to the same group keep together while walking. And the inter-group interaction was introduced so as not to collide with persons in the different groups. Grouping behavior model was validated directly by comparison with the footage. The development is reasonably successful in simulating grouping behavior. However, further enhancement need to be performed by validating with the footage with a top view setting at the field. The validated model is useful to replicate crowd dynamics in consideration of grouping behavior and have significant usage in developing reliable prediction models for the design of urban infrastructure, traffic management, and crowd safety during mass events or evacuation process.

\section{ACKNOWLEDGEMENT}

This work was done with the collaboration with Laboratory of Hitoshi Gotoh, Kyoto University and Universiti Sains Malaysia (USM).

\section{REFERENCES}

[1] Statistica, 2015. Malaysia: Urbanization from 2004 to 2015. Retrieved from https://www.statista.com/statistics/455880/ urbanization-in-malaysia/.

[2] Moussaid, M., Perozo, N., Garnier, S., Helbing, D., and Theraulaz, G. 2010. The Walking Behaviour of Pedestrian Social Groups and Its Impact on Crowd Dynamics. PLoS ONE, 5 (4), e10047. doi: 10.1371/ journal.pone.0010047.

[3] Sarmady, S., Haron, F., and Talib, A. Z. 2009. Modeling Groups of Pedestrian in Least Effort Crowd Movements using Cellular Automata. In 2009 Third International Conference on Modeling and Simulation doi: 10.1109/AMS.2009.16.

[4] Qiu, F., and Hu, X. 2010. Modeling Group Structures in Pedestrian Crowd Simulation. Simulation Modelling Practice and Theory, 18, 190-205. doi: 10.1016/j.simpat.2009.10.005.

[5] Gotoh, H., Harada, E., and Andoh, E., 2012. Simulation of Pedestrian Contra-Flow by Multi-Agent DEM Model with Self-Evasive Action Model. Safety Science, 50, 326-332. doi 10.1016/j.ssci.2011.09.009

[6] Harada, E., Gotoh, H., and Abd Rahman, N., 2015. A Switching Action Model for DEM-based Multi Agent Crowded Behavior Simulator. Safety Science, 79, 105-115. doi 10.1016/j.ssci.2015.06.001. 